\title{
Modulating Arm Swing Symmetry with Cognitive Load: A Window on Rhythmic Spinal Locomotor Networks in Humans?
}

\author{
Tim Killeen, ${ }^{1}$ Christopher S. Easthope, Linard Filli, ${ }^{2}$ Michael Linnebank, ${ }^{2,3}$ Armin Curt, \\ Marc Bolliger, and Björn Zörner ${ }^{1}$
}

\begin{abstract}
In healthy subjects, changes in arm swing symmetry while walking are observed when a cognitive dual task is added, with a tendency toward left-dominant arm swing as cognitive load increases. We applied a modified Stroop word/color naming paradigm to investigate this effect in spinal cord injured (SCI) patients. Six patients with cervical SCI (cSCI), 6 with thoracic injuries (tSCI; all 12 patients American Spinal Injury Association [ASIA] Injury Score [AIS]D), and 12 healthy, matched controls underwent three-dimensional 3D gait analysis while walking normally at a comfortable speed (NW) and when performing an additional congruent (CS) and incongruent (IS) Stroop task. An arm swing symmetry index (ASI) -in which positive values indicate proportionally more movement on the left and vice versa-was calculated. Even in the baseline NW condition, all three subject groups showed larger arm movements on the left. In controls, ASI increased (NW, 13.7 \pm 6.3 ; CS, 16.6 \pm 6.4 ; IS, 19.6 \pm 7.8 ) as the task became more demanding. A larger shift in tSCI patients (NW, $15.8 \pm 6.0$; CS, $23.4 \pm 3.8$; IS, $30.7 \pm 4.4)$ was driven by a significant reduction in right wrist trajectory $(p=0.014)$, whereas cSCI patients showed a small reduction in mean ASI with high variability (NW, 14.2 \pm 10.7 ; CS, 9.3 \pm 13.5 ; IS, $6.0 \pm 12.9$ ). The effect of the IS task on ASI compared to baseline (NW) was significantly different between tSCI $(+12.5 \pm 6.3)$ and cSCI $(-8.2 \pm 6.0)$ patients $(p=0.011)$. Disruption of the long propriospinal connections coordinating arm and leg movements during walking may explain the heightened sensitivity to manipulation of cognitive load in tSCI, whereas the more robust automaticity in cSCI may be attributed to impaired supraspinal inputs in the context of preserved intraspinal pathways.
\end{abstract}

Keywords: arm swing; dual-task; motor control; spinal cord injury; symmetry

\section{Introduction}

A SYMMETRY of the rhythmic upper limb movements during ambulation has been identified as a potentially useful diagnostic indicator of impaired motor control. ${ }^{1,2}$ In healthy individuals, arm swing asymmetry has recently been observed to increase while engaging in cognitive dual task during walking. ${ }^{3,4}$ This increase in asymmetry may be directional, with increasing cognitive load in the Stroop word/color discrimination task causing asymmetry to shift toward proportionally smaller arm swing amplitudes on the right. ${ }^{4}$ Why this effect should be lateralized in this way is not entirely clear, but its evaluation in spinal cord injury (SCI) patients may present a useful method to investigate motor control of the upper limbs during ambulation.

The upper and lower limbs in human locomotion are thought to be coordinated by bidirectional propriospinal fibers coupling lumbar and cervical spinal locomotor circuits, networks capable of producing coordinated, rhythmic movements modulated by task and cycle phase as well as a degree of supraspinal influence. ${ }^{5-7}$ The cervical component of the human networks affecting arm swing is thought to reside at level of the cervical enlargement, with inputs from the lower-limb circuits in the lumbar enlargement conducted along long propriospinal pathways and through local segmental propagation of rhythmicity in the thoracic cord..$^{8-12}$ It may therefore be hypothesized that an incomplete injury rostral to the cervical circuits will render arm swing less sensitive to changes in supraspinal drive arising from dual-task performance, whereas a thoracic injury partially disrupting intersegmental networks would result in heightened sensitivity to cognitive modulation. We employed an established dual-task paradigm based on the Stroop task in patients with incomplete thoracic (tSCI) or cervical $\mathrm{SCI}$ (cSCI) and matched controls to assess the changes in arm

\footnotetext{
${ }^{1}$ Spinal Cord Injury Center, University Hospital Balgrist, Zurich, Switzerland.

${ }^{2}$ Department of Neurology, University Hospital Zurich, Zurich, Switzerland.

${ }^{3}$ Department of Neurology, Helios-Klinik Hagen-Ambrock, Hagen, Germany.

$\mathrm{MB}$ and $\mathrm{BZ}$ contributed equally to the article.
} 
swing asymmetry brought about by increases in cognitive load while walking.

\section{Methods}

This study, approved by the cantonal ethics committee of Zurich (KEK-2014/0004), was carried out at Balgrist University Hospital in accord with Good Clinical Practice and the Declaration of Helsinki. Patients with SCI able to walk on a horizontal treadmill without handrail support were consecutively recruited by mailed flyers and during hospital visits. To ensure they could walk at a speed at which stable 1:1 arm swing movements are usually produced, ${ }^{13}$ all patients were required to demonstrate a maximal overground walking speed of at least $3.5 \mathrm{kmh}^{-1}$ as measured during the $10-\mathrm{m}$ walk test (10MWT). Controls were drawn from a pool of healthy subjects who had previously undergone an identical protocol and matched 1:1 with patients based on age, sex, height, and weight. All participants gave informed written consent.

At a first session, subjects were screened for any significant orthopedic, neurological, cardiac, or pulmonary abnormality other than the existing SCI in patients. Color-blind participants were also excluded. Patients underwent a full neurologic assessment, including American Spinal Injury Association impairment scale (AIS) scoring. ${ }^{14}$ Patients were grouped as cervical or thoracic based on whether or not the lowest component of their cord lesion on magnetic resonance imaging was below the anatomical C8 level. Included subjects then underwent a 40-min acclimatization protocol on the treadmill, which included four, 45 -sec rehearsals of the different dual-task conditions. Subjects were blinded to the purpose of the study.

Within 7 days, participants returned for full-body gait analysis. The $10 \mathrm{MWT}^{15}$ and the timed 25 -foot walk $(25 \mathrm{FWT})^{16}$ were performed. Mean maximal overground speed was calculated from the mean of two 25FWT attempts. Three-dimensional (3D) gait analysis was conducted while walking on an instrumented treadmill $(120 \mathrm{~Hz}$; FDM-T; Zebris Medical GmbH, Isny im Allgäu, Germany) in a clinical gait laboratory equipped with 10 infrared cameras using Nexus software (version 1.8.5; Vicon Motion Systems Ltd, Oxford, UK) at a sampling rate of $200 \mathrm{~Hz}$. An extended full-body Plug-in-Gait marker set (Vicon Motion Systems Ltd) was applied to the participants for motion tracking.

Subjects performed all trials barefoot without handrail support on the treadmill at $50 \%$ of their mean maximal overground walking speed as assessed in the 25FWT. ${ }^{17}$ Participants were asked to fixate on a black cross displayed at eye height in the middle of a monitor positioned in front of the treadmill. Trials were repeated if the subject was observed to touch the handrails or make undesired, goal-directed arm movements (e.g., nose scratching, adjusting hair). Stable gait was recorded over a period of $45 \mathrm{sec}$.

Cognitive load was added using a modified Stroop paradigm. ${ }^{18,19}$ In place of the black cross displayed during the normal walking tasks, text spelling out four colors (red, green, yellow, and blue) was presented at pseudorandom intervals using Powerpoint 2010 (Microsoft Corp., Redmond, WA). The duration of presentation of each stimulus was between 600 and $1400 \mathrm{~ms}$ (mean, $1 \mathrm{~Hz}$ ). The task was presented in the participant's self-declared native language and script. In the congruent Stroop condition, the coloring of the text was consistent with that of the color spelled out in the text. In the incongruent condition, all stimuli consisted of spelled colors presented in one of the four index colors not corresponding to the written stimulus.

Raw data were reconstructed and labeled semiautomatically in Nexus. Lower-body gait cycle events were set using data exported from the force plate under the treadmill. Arm swing cycle was defined by the maximal protraction and retraction of the wrist joint center. Final data were then screened in ProCalc 1.1 (Vicon Motion Systems Ltd) for undesired arm movements by identifying cycles in which the wrist center was raised above the level of the sternum in the vertical axis. Cycles including such movements and the preceding and subsequent three cycles were removed from the analysis. Final data were processed using ProCalc to output spatiotemporal gait parameters for all trials.

The 3D trajectory of a point half way between the two wrist markers was the principal measure of arm swing. ${ }^{1}$ The arm swing symmetry index (ASI) is expressed as follows ${ }^{4,20}$;

$$
A S I=\left(\frac{L-R}{\max (L, R)}\right) \times 100
$$

in which $L$ is the wrist trajectory on the left and $R$ that on the right, giving an index value between -100 and 100 , with 0 representing perfectly symmetrical movements and trials in which the wrist trajectory was longer on the right than the left yielding a negative index and vice versa.

Differences in demographic data between groups were assessed for statistical significance using Wilcoxon signed-rank tests. ASI values for each group were analyzed statistically using a nonparametric Friedman analysis in which condition was a repeated measure. A Kruskal-Wallis test was used to assess differences across the three groups. When these returned significant results, pair-wise post-hoc Wilcoxon signed-rank tests were performed with Dunn-Bonferroni correction for multiple comparisons. To look for symmetry effects in the lower limbs, symmetry indices for left and right knee range of motion (ROM) and step length were calculated and analyzed using the same approach as for ASI. Relationships between ASI during normal walking and ASI change under the two dual-task conditions with age, sex, time post-injury, asymmetry of upper-limb impairment (calculated from the left and right upper extremity motor scores [UEMS] in the same manner as the ASI) were assessed using Spearman's rho and likewise corrected for multiple comparisons. Statistical significance was set at $p \leq 0.05$ for all tests.

\section{Results}

Six patients with a cervical SCI ( 1 female), 6 with a thoracic SCI (2 female), and 12 matched control participants were recruited. Patient and control subject characteristics are summarized in Table 1. Although mean UEMS was lower in the cSCI group (48.5 vs. 50), this difference did not reach significance ( $p=0.059)$. Likewise, there were no significant differences between the groups in terms of lower extremity motor scores (LEMS), UEMS and LEMS laterality, other clinical scores, or time post-injury; all patients were classified as AIS D. Patient groups walked on the treadmill at similar median speeds (set as $50 \%$ of their maximal overground speed: cervical patients, $3.1 \mathrm{kmh}^{-1}$; interquartile range [IQR], 1.22; thoracic patients, $2.95 \mathrm{kmh}^{-1}$; IQR, 1.05), which was significantly slower than the controls ( $4.0 \mathrm{kmh}^{-1}$; IQR, $\left.1.22 ; p \leq 0.000\right)$.

All patients and control subjects exhibited one arm swing cycle per lower-limb gait cycle during normal treadmill walking. During normal walking, the length of the mean wrist trajectories on the left and the right during normal walking were not significantly different between the control and either of the patient groups (Fig. 1). In all three walking conditions, left arm wrist trajectory was consistently larger than that on the right, and this discrepancy became significant in the tSCI group under the incongruent Stroop task $(p=0.014)$.

Accordingly, mean ( \pm standard error of the mean) ASI during normal walking was $13.7 \pm 6.3,14.2 \pm 10.7$, and $15.8 \pm 6.0$ in controls, cervical, and thoracic patients, respectively, indicating arm swing movements that were proportionally smaller on the right. These values increased to $16.6 \pm 6.4$ and $23.4 \pm 3.8$ in controls and 


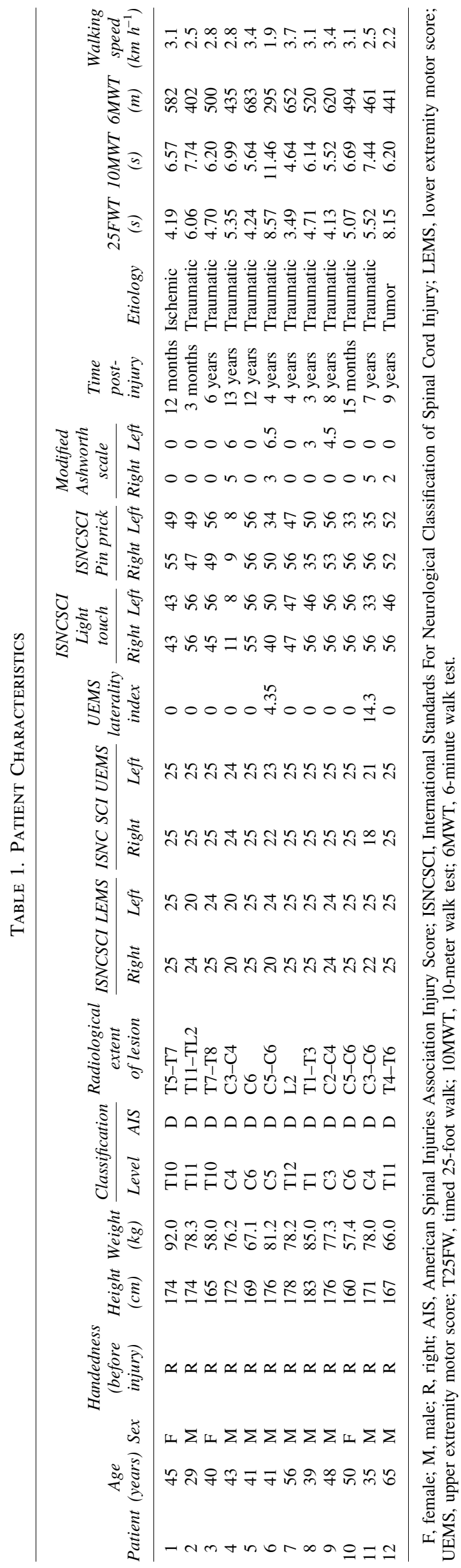




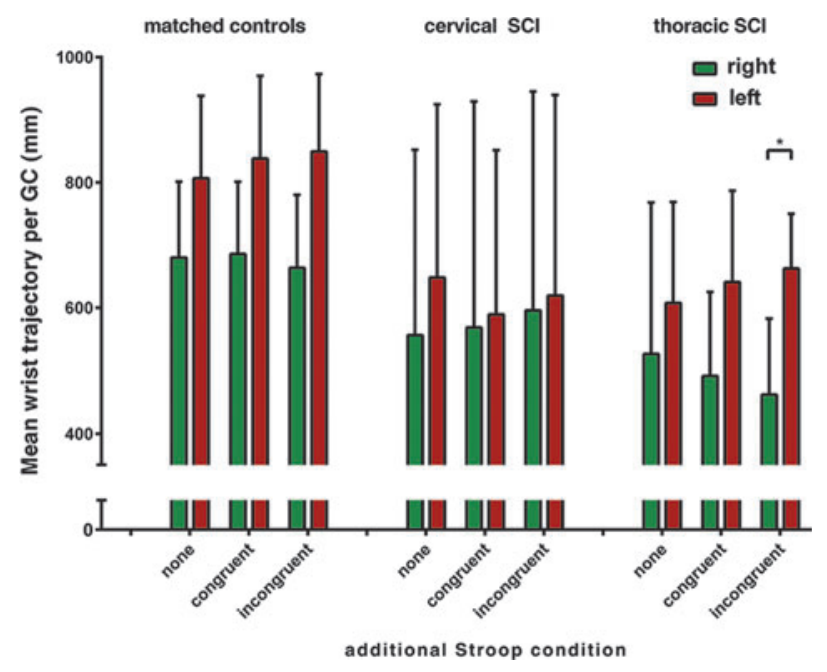

FIG. 1. Absolute wrist trajectory length. Mean threedimensional wrist center trajectories for 6 patients with cervical SCI, 6 with thoracic SCI, and 12 matched controls under increasing levels of cognitive load in a treadmill dual task. Error bars indicate standard deviation. Statistical significance was determined using a repeated-measures Friedman analysis with posthoc Wilcoxon rank-sum tests. $p$ values were corrected for multiple comparisons within each group using the Dunn-Bonferroni method. GC, gait cycle; SCI, spinal cord injury. Color image is available online at www.liebertpub.com/neu

thoracic patients during the congruent Stroop task. In both these groups, the performance of the incongruent Stroop task was associated with a further increase, to $19.6 \pm 7.8$ in controls and $30.7 \pm 4.4$ in thoracic patients. In contrast, mean ASI in cervical patients decreased to $9.3 \pm 13.5$ and $6.0 \pm 12.9$ in the congruent and incongruent Stroop tasks, respectively (Fig. 2). No significant differences were found in either of the lower-limb symmetry indices (knee ROM and step length) across the three conditions.

The relative effect of the concurrent Stroop tasks on the ASI during gait was evaluated. Control subjects showed a modest increase (i.e., toward further left-sided dominance of arm swing movements) from the baseline normal walking task of $2.4 \pm 3.5$ in the congruent Stroop task and subsequently to $6.5 \pm 3.8$ during the incongruent task (Fig. 3). Equivalent values in the cervical group were $-4.8 \pm 3.9$ and $-8.2 \pm 6.0$, whereas the decrease in right arm swing in the thoracic group resulted in ASI changes of $8.4 \pm 4.2$ and $12.5 \pm 6.3$ in this group. The changes in ASI brought about by the incongruent Stroop task in the cervical and thoracic groups were significantly different $(p=0.011)$.

\section{Discussion}

Recently, it has been shown that symmetry of arm movements during gait can be modulated during cognitive dual tasks, ${ }^{3}$ particularly the Stoop task. ${ }^{4}$ A paucity of arm swing on the right may be accounted for by reallocation of supraspinal resources during dual tasking away from the maintenance of arm swing; it is pronounced in older adults, in whom cognitive resources are degraded. ${ }^{3,4}$ Precisely why the right side is more sensitive to this effect is unclear, but may be attributed to a reduction in corticospinal drive resulting either from interference stemming predominantly from left hemisphere language processing, differential degrees of connectivity to the dominant and nondominant arms, heightened sensitivity to interference within the dominant hemisphere, or a combination of

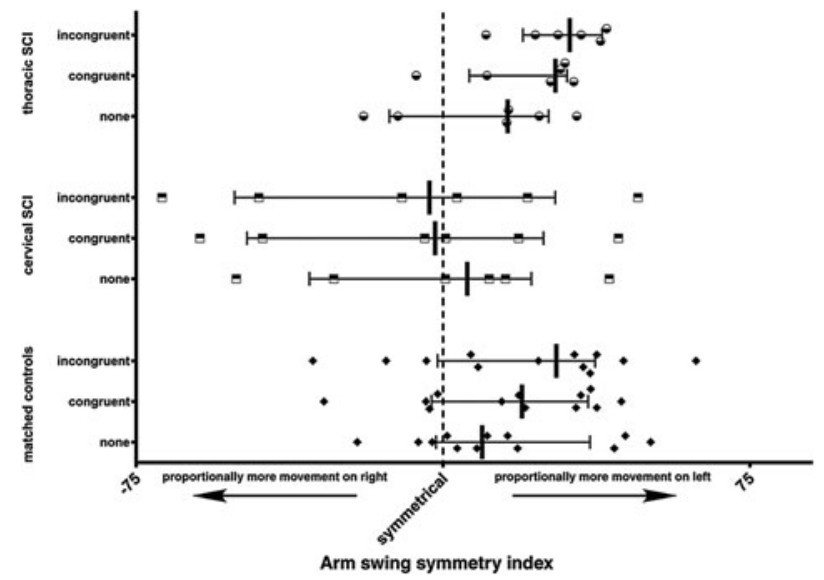

FIG. 2. Arm swing symmetry index (ASI) under increasing cognitive load. Wrist trajectory symmetry index is calculated using the left and right three-dimensional wrist center trajectories, with left dominance resulting in a positive value and vice versa. ASI is given as the mean value per gait cycle over a trial of $45 \mathrm{sec}$ (approximately 34 gait cycles at $3.1 \mathrm{kmh}^{-1}$ ). In the trial with no additional task, participants looked directly ahead at a black cross in the center of a screen placed at eye height. Median ASI with 95\% confidence intervals is shown, with individual ASI results indicated for each condition and subject group. SCI, spinal cord injury.

these factors. All our patients and controls were right-handed, precluding testing of hypotheses related to language hemisphere dominance. A previous report examined the effect of handedness on ASI in a small cohort and under varying locomotor (but not dualtask) conditions and concluded that ASI was not related to handedness, ${ }^{20}$ although given that the majority of left-handers and virtually all right-handers have language centers in the left hemisphere, further study in truly right-lateralized individuals or larger samples may be more revealing.

Arm swing has been relatively neglected as a feature of ambulatory behavior in patients with incomplete SCI (iSCI). The most thorough studies of upper-limb kinematics in iSCI aimed to

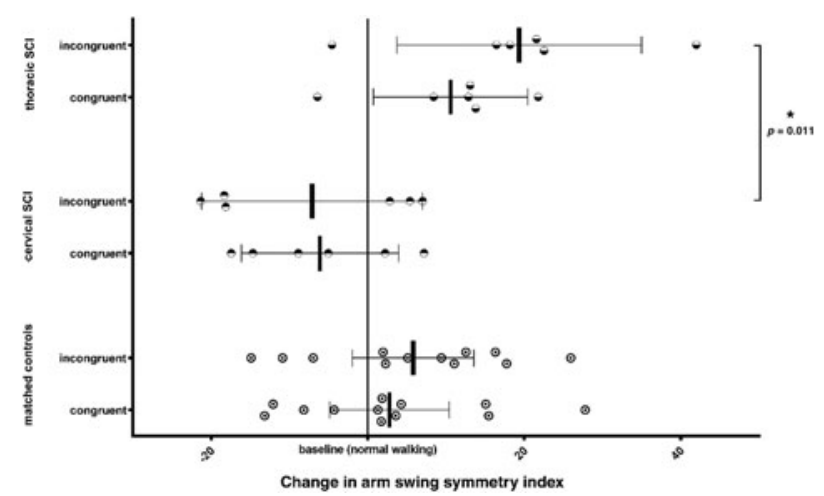

FIG. 3. Change in arm swing symmetry index (ASI) under congruent and incongruent Stroop tasks. Median ASI change with 95\% confidence intervals show deviation from baseline ASI recorded during normal walking. Statistical significance was determined using a Kruskal-Wallis analysis with post-hoc Wilcoxon rank-sum tests. $p$ values were corrected for multiple comparisons within each group using the Dunn-Bonferroni method. SCI, spinal cord injury. 
establish the presence or absence of arm swing in iSCI. These rather heterogeneous samples included relatively poor walkers in whom arm swing deficits may have been significantly affected by lateralized pathology, spasticity, or habituated use of walking aids. ${ }^{21,22}$ In order to investigate the modulation of arm swing asymmetry in the context of spinal lesions, we attempted to recruit a morehomogenous group of patients who, despite their iSCI, were able to produce near-normal walking patterns, including bilateral arm swinging, on the treadmill at a comfortable walking speed. The patient groups were closely matched, with median speed and wrist trajectory and clinical and demographic parameters similar in cervical and thoracic patients (Table 1).

Control participants exhibited a trend toward more leftdominant arm swing under increasing cognitive load against a background of significant variability, in keeping with previous studies in healthy subjects. ${ }^{3,4,20}$ Cervical patients showed no such leftward tendency, and wrist trajectories remained essentially the same on the left and right under all three conditions. Patients with thoracic lesions, however, showed a considerably morehomogenous response to the Stroop tasks than the other groups, driven by the significant reductions in arm swing on the right side.

These findings fit well with the current understanding of interlimb coordination in humans, in which locomotor patterns emanating from the lumbar enlargement are coupled by long propriospinal and short intersegmental pathways with their cervical counterpart circuits, with the latter functionally subservient to the former. ${ }^{12}$ Disruption of these thoracic pathways in patients with lesions below $\mathrm{T} 1$ may result in an adaptive, greater supraspinal role in the maintenance of arm swing, with right arm swing thus significantly more sensitive to cognitive interference from a left hemisphere dual task. Interpretation is more complicated in our cohort of cervical patients, of whom 4 had lesions that extended below $\mathrm{C} 4$, potentially directly disrupting the motor pools projecting to the arm muscles. It is therefore not possible to conclude with certainty what underlies the lack of a coherent response to cognitive modulation observed in the cervical patients, although they certainly do not exhibit the homogenous behavior of those with thoracic injuries. The 2 patients with lesions unequivocally cranial to the cervical enlargement showed heterogeneous responses to cognitive load (individual patient data not shown), with 1 patient (sub-C4) demonstrating a small shift toward left dominant swing with the incongruent Stroop task. The other (sub-C3) showed a shift from a moderately left-dominant swing trajectory during $\mathrm{NW}$ to a right-dominant one of similar magnitude during the incongruent Stroop task.

This study is limited by its small sample size and preliminary nature. Future studies should include the use of upper- and lowerlimb electromyography to better characterize what drives the reduction in right arm swing observed in patients with thoracic injuries. By excluding patients unable to walk on the treadmill without handrail support and therefore produce arm swing movements, we may have selected a patient group in whom sensory symptoms were disproportionally dominant, an assumption supported by the motor and sensory scores in Table 1 . In vertebrates, the propriospinal fibers thought to be responsible for interlimb coordination are scattered radially throughout the white matter of the cord with fiber density increasing toward the central gray matter. ${ }^{23}$ With reference to current knowledge of human propriospinal anatomy, we see no reason why patients with more dorsal injuries should be more or less susceptible to the effects observed in this study, although a larger cohort with lesion characterization may allow investigation of this question.
We used relative speed matching between the control and patient groups. Walking speed on the treadmill was based on each individual's maximal overground walking speed, used as an outcome measure to quantify overall walking function in the clinical setting ${ }^{17}$ and at which the automaticity of arm swing is likely to be strongest. Thus, healthy subjects, as well as patients with different degrees of walking dysfunctions, walked at a speed that was proportional to their walking ability and which was perceived as comfortable by all participants. Although this objective method of speed definition is advantageous in challenging participants with dissimilar walking dysfunction to a proportional degree, different absolute walking speeds may affect arm swing symmetry. This aspect was briefly addressed by Plate and colleagues, who observed that, although absolute, bilateral arm swing amplitude increases with speed, ASI is preserved across a range of walking speeds. ${ }^{4}$

As others have done, ${ }^{24}$ we used two levels of Stroop task in an attempt to demonstrate a rudimentary dose-response relationship to the increasing cognitive demands of the two tasks. Although a trend in this direction was observed in all three groups studied, these did not reach significance. This may be representative of statistical underpowering for the detection of a specific difference between the degrees of Stroop difficulty. Alternatively, the site(s) of cognitivemotor interference eliciting the reduction in right arm swing may be common to both versions of the Stroop task used in this study.

Both the patient and control groups exhibited a degree of arm swing asymmetry during normal walking. Whereas such spontaneous asymmetry may be explained in certain cervical patients as a consequence of an asymmetrical injury, its marked presence in healthy controls and thoracic patients is more difficult to account for. Moreover, the degree and direction of baseline asymmetry in the patients with relatively mild cervical injuries here was not correlated with lateralized UEMS. This inherent variability in ASI has implications for the usefulness of arm swing asymmetry as a diagnostic parameter or clinical trials outcome measure, although within subjects, the use of cognitive load modulation during walking appears to be a potential method for estimating the degree of supraspinal contribution to arm swing. All subjects received an equal amount of acclimatization performing the two Stroop tasks, standardizing task novelty. As such, we cannot exclude the possibility that task familiarity may attenuate the observed shift in ASI as participants gain exposure, although ASI was generally quite stable throughout the individual 45 -sec trials.

Refinement of both the dual-task methodology used and perhaps the gait parameters used to define arm swing and ASI may lead to a clinical or research application of this interesting phenomenon.

\section{Acknowledgments}

This study was supported by the Clinical Research Priority Program for NeuroRehab of the University of Zurich. The authors thank Lilla Lörincz, MSc, for her hard work and professionalism in the gait lab. The authors also acknowledge the invaluable contribution of the patients and control subjects who volunteered to take part in the study.

\section{Author Disclosure Statement}

No competing financial interests exist.

\section{References}

1. Lewek, M.D., Poole, R., Johnson, J., Halawa, O., and Huang, X. (2010). Arm swing magnitude and asymmetry during gait in the early stages of Parkinson's disease. Gait Posture 31, 256-260. 
2. Roggendorf, J., Chen, S., Baudrexel, S., van de Loo, S., Seifried, C. and Hilker, R. (2012). Arm swing asymmetry in Parkinson's disease measured with ultrasound based motion analysis during treadmill gait. Gait Posture 35, 116-120.

3. Mirelman, A., Bernad-Elazari, H., Nobel, T., Thaler, A., Peruzzi, A., Plotnik, M., Giladi, N., and Hausdorff, J.M. (2015). Effects of aging on arm swing during gait: the role of gait speed and dual tasking. PLoS One 10, e0136043.

4. Plate, A., Sedunko, D., Pelykh, O., Schlick, C., Ilmberger, J.R., and Bötzel, K. (2015). Normative data for arm swing asymmetry: how (a)symmetrical are we? Gait Posture 41, 13-18.

5. Duysens, J., Tax, A.A., Trippel, M., and Dietz, V. (1992). Phasedependent reversal of reflexly induced movements during human gait. Exp. Brain Res. 90, 404-414.

6. Dietz, V. (2003). Spinal cord pattern generators for locomotion. Clin. Neurophysiol. 114, 1379-1389.

7. Barthelemy, D., and Nielsen, J.B. (2010). Corticospinal contribution to arm muscle activity during human walking. J. Physiol. 588, 967-979.

8. Dietz, V. (2011). Quadrupedal coordination of bipedal gait: Implications for movement disorders. J. Neurol. 258, 1406-1412.

9. Nathan, P.W., Smith, M., and Deacon, P. (1996). Vestibulospinal, reticulospinal and descending propriospinal nerve fibres in man. Brain 119, 1809-1833.

10. Zehr, E.P., Carroll, T.J., Chua, R., Collins, D.F., Frigon, A., Haridas, C., Hundza, S.R., and Thompson, A.K. (2004). Possible contributions of CPG activity to the control of rhythmic human arm movement. Can. J. Physiol. Pharmacol. 82, 556-568.

11. Solopova, I.A., Selionov, V.A., Zhvansky, D.S., Gurfinkel, V.S., and Ivanenko, Y.P. (2016). Human cervical spinal cord circuitry activated by tonic input can generate rhythmic arm movements. J. Neurophysiol. 115, 1018-1030.

12. Falgairolle, M., de Seze, M., Juvin, L., Morin, D., and Cazalets, J.R. (2006). Coordinated network functioning in the spinal cord: an evolutionary perspective. J. Physiol. Paris 100, 304-316.

13. Frykberg, G.E., Johansson, G.M., Schelin, L., and Häger, C.K. (2014). The Arm Posture Score for assessing arm swing during gait: an evaluation of adding rotational components and the effect of different gait speeds. Gait Posture 40, 64-69.

14. Kirshblum, S.C., Burns, S.P., Biering-Sorensen, F., Donovan, W., Graves, D.E., Jha, A., Johansen, M., Jones, L., Krassioukov, A. Mulcahey, M., Schmidt-Read, M., and Waring, W. (2011). International standards for neurological classification of spinal cord injury (revised 2011). J. Spinal Cord Med. 34, 535-546.
15. Rossier, P., and Wade, D.T. (2001). Validity and reliability comparison of 4 mobility measures in patients presenting with neurologic impairment. Arch. Phys. Med. Rehabil. 82, 9-13.

16. Bohannon, R. (1997). Comfortable and maximum walking speed of adults aged 20-79 years: reference values and determinants. Age Ageing 26, 15-19.

17. Zörner, B., Filli, L., Reuter, K., Kapitza, S., Lörincz, L., Sutter, T., Weller, D., Farkas, M., Easthope, C.S., Czaplinski, A., Weller, M., and Linnebank, M. (2016). Prolonged-release fampridine in multiple sclerosis: Improved ambulation effected by changes in walking pattern. Mult. Scler. Jan 13. pii: 1352458515622695. [Epub ahead of print]

18. Stroop, J. (1935). Studies of interference in serial verbal reactions. J. Exp. Psychol. 121, 15-23.

19. MacLeod, C.M. (1991). Half a century of research on the Stroop effect: an integrative review. Psychol. Bull. 109, 163-203.

20. Kuhtz-Buschbeck, J.P., Brockmann, K., Gilster, R., Koch, A., and Stolze, H. (2008). Asymmetry of arm-swing not related to handedness. Gait Posture 27, 447-454.

21. Tester, N.J., Barbeau, H., Howland, D.R., Cantrell, A., and Behrman, A.L. (2012). Arm and leg coordination during treadmill walking in individuals with motor incomplete spinal cord injury: a preliminary study. Gait Posture 36, 49-55.

22. Tester, N.J., Howland, D.R., Day, K. V, Suter, S.P., Cantrell, A., and Behrman, A.L. (2011). Device use, locomotor training, and the presence of arm swing during treadmill walking post-spinal cord injury. Spinal Cord 49, 451-456.

23. Filli, L., Engmann, A.K., Zorner, B., Weinmann, O., Moraitis, T., Gullo, M., Kasper, H., Schneider, R., and Schwab, M.E. (2014). Bridging the gap: a reticulo-propriospinal detour bypassing an incomplete spinal cord injury. J. Neurosci. 34, 13399-13410.

24. Doi, T., Asai, T., Hirata, S., and Ando, H. (2011). Dual-task costs for whole trunk movement during gait. Gait Posture 33, 712-714.

Address correspondence to:

Tim Killeen, MRCS

Spinal Cord Injury Center

University Hospital Balgrist

Forchstrasse 340

8008 Zurich, Switzerland

E-mail: tim.killeen@balgrist.ch 